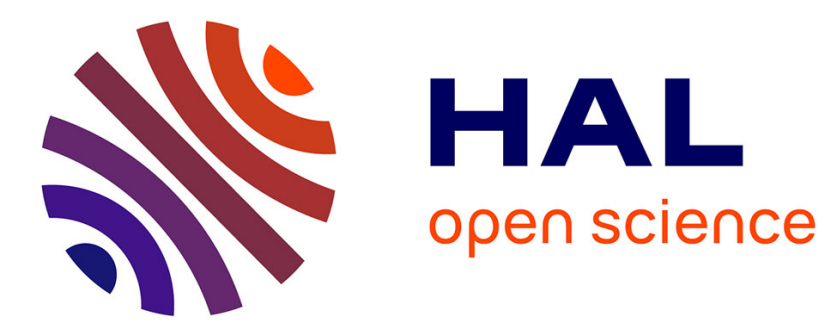

\title{
Rapports des usagers au subutex: de la reconquête de l'autonomie à la spirale de l'échec
}

Anne Guichard, France Lert, Jean-Marc Brodeur, Lucie Richard

\section{To cite this version:}

Anne Guichard, France Lert, Jean-Marc Brodeur, Lucie Richard. Rapports des usagers au subutex: de la reconquête de l'autonomie à la spirale de l'échec. Sciences Sociales et Santé, 2006, 24 (4), pp.5-43. inserm-00141346

\section{HAL Id: inserm-00141346 https://www.hal.inserm.fr/inserm-00141346}

Submitted on 12 Apr 2007

HAL is a multi-disciplinary open access archive for the deposit and dissemination of scientific research documents, whether they are published or not. The documents may come from teaching and research institutions in France or abroad, or from public or private research centers.
L'archive ouverte pluridisciplinaire HAL, est destinée au dépôt et à la diffusion de documents scientifiques de niveau recherche, publiés ou non, émanant des établissements d'enseignement et de recherche français ou étrangers, des laboratoires publics ou privés. 
RAPPORTS DES USAGERS AU SUBUTEX@: DE LA RECONQUETE DE L'AUTONOMIE A LA SPIRALE

DE L'ECHEC.

\author{
Anne GUICHARD * \\ France LERT* \\ Jean-Marc BRODEUR** \\ Lucie, RICHARD**
}

Mots clés: toxicomanie, traitement de substitution, Subutex ${ }^{\circledR}$, usages, perspective de l'usager

Cette étude a été financée par la Mission interministérielle de lutte contre la drogue et la toxicomanie) (MILDT) et l'ANRS (Agence Nationale de recherche sur le Sida)

* Anne Guichard, doctorante en Santé publique, Hôpital National de Saint-Maurice, Inserm, U687, IFR69, 14, rue du Val d'Osne, Saint-Maurice, F-94415 France.

email : aguichard@netcourrier.com

* France LERT*, directrice de recherche, Inserm, U687, IFR69, 14, rue du Val d'Osne, SaintMaurice, F-94415 France.

** Jean-Marc Brodeur, Professeur titulaire, Groupe de Recherche Interdisciplinaire en Santé, Montréal (GRIS), Université de Montréal, Département de médecine sociale et préventive, Qc, Canada

** Lucie Richard, Professeur titulaire, Groupe de Recherche Interdisciplinaire en Santé, Montréal (GRIS), Université de Montréal, Département des Sciences Infirmières, Qc, Canada 


\section{REMERCIEMENTS}

La conduite de cette étude a été rendue possible grâce à l'implication des intervenants des structures partenaires (CSST, Cabinets libéraux et PES). Les auteurs souhaitent remercier et exprimer leur reconnaissance à toutes les personnes ayant participé à l'enquête, en particulier celles qui se sont rendues disponibles et se sont investies dans la durée des trois entretiens, ainsi que Christine Calderon qui a retranscrit tous les entretiens. 
Résumé : Les traitements de substitution se sont imposés en France comme stratégie de prise en charge des personnes dépendant de l'héroïne dans le contexte de l'infection VIH/sida. Les modalités choisies en France pour diffuser ce modèle, en particulier le choix de la buprénorphine et le rôle majeur des médecins de ville, tranchent avec le caractère strict de leur mise à disposition dans d'autres pays et laissent place à des usages autonomes pour partie intégrés aux drogues de rue qui conduisent à une mise en cause du bien-fondé de cette stratégie. Une enquête par entretiens répétés a été conduite auprès d'un échantillon diversifié de 28 personnes en traitement. L'analyse a permis de dégager quatre profils illustrant le rapport des usagers au produit de substitution. Ces profils conjuguent les effets recherchés ou non de la molécule, la prise du médicament selon des règles auto-définies ou dans le strict respect de la prescription, la transformation du vécu de la toxicomanie dans le sens d'une reprise de contrôle sur les consommations ou d'une perte de contrôle aggravée, les ressources sociales et personnelles ou leur absence. La représentation péjorative du toxicomane continue à peser sur tous, faisant pour les uns obstacle à la reconstruction de l'image de soi, contribuant à une dévalorisation accrue pour les autres.

Abstract: In France, in the context of HIV-AIDS epidemics, substitution treatment emerged as the standard for heroin addiction treatment. The French model of substitution treatment buprenorphine, prescription by GPs and delivery in community pharmacies - contrasts with the restrictive regulation in many other countries. This flexible framework allows for substitution drug use out of regular guidelines and questions the validity of such a strategy. A qualitative study based on repeated interviews with a convenient sample of 28 drug users under buprenorphine was carried out to draw patterns of substitution treatment use and misuse. Four profiles are identified combining desired or adverse effects of the buprenorphine pills, self defined regimens for taking prescription drugs, improvement or worsening in dependence, existence or lack of social and personal resources. The negative image of the IV drug user hangs over all individuals under substitution treatment and hinders the construction of a new self-image. 


\section{INTRODUCTION}

Avec l'émergence du sida, les traitements de substitution pour la dépendance à l'héroïne sont progressivement passés d'une stricte perspective thérapeutique à une mesure de santé publique inscrite au sein du paradigme de réduction des risques. Pour autant, la mise en place de programmes de prévention (les programmes d'échange de seringues -PES-, par exemple) et la normalisation du traitement ne font pas des usagers de drogues des citoyens et des malades comme les autres. Leur point de vue reste aujourd'hui encore largement négligé dans l'élaboration des politiques publiques et les recherches portant sur la prise en charge des addictions toujours dominés par la perspective médicale et épidémiologique (Montagne, 1998). Dans un domaine soumis à des enjeux idéologiques et moraux aussi puissants que celui des «drogues » et de la «toxicomanie », notre étude s'intéresse à l'expérience et aux pratiques concrètes des usagers qui utilisent ces produits de substitution sur de très longues périodes, pour mieux comprendre les logiques qui sous tendent les usages et la façon dont elles se construisent dans la vie quotidienne.

La dépendance tend aujourd'hui à être assimilée à une condition chronique nécessitant une prise en charge de très longue durée (Newman, 1996). Le principe qui sous-tend les traitements de substitution consiste, à partir de la prescription médicale d'un produit qui soulage le manque sans produire de sensations agréables et d'une prise en charge psychosociale adaptée, à offrir un cadre de vie sorti de l'illégalité pour permettre à la personne de réduire (voire d'arrêter) ses consommations de drogues et les risques associés, de s'occuper de sa santé, et d'adopter un mode de vie moins déviant, notamment par l'insertion professionnelle et l'arrêt de la délinquance.

Aujourd'hui encore, les traitements de substitution se réfèrent principalement au champ de la recherche clinique (Montagne, 2002) qui met en jeu des méthodes d'évaluation classiques de l'efficacité des traitements (essais thérapeutiques et cohortes observationnelles) utilisant des indicateurs cliniques, pharmacologiques, comportementaux, ou de santé publique. La façon dont 
ces produits de substitution s'intègrent dans le quotidien des usagers reste peu documentée (Agar et al., 2001). Quelques études ont cependant été réalisées en Angleterre, aux Etats-Unis, au Canada, en Australie et aux Pays Bas. Les données issues de ces études qualitatives menées auprès d'usagers sous méthadone indiquent des motivations variées et des bénéfices nuancés. La revue de la littérature montre que la prise de la méthadone correspond à des situations très hétérogènes. Entre la volonté de retrouver un mode de vie plus ordinaire, de rompre avec les substances illicites, de soulager la douleur répétitive du manque et de réduire les risques (sociaux et sanitaires), la prise de méthadone peut également offrir une opportunité économique sous forme d'accès à des allocations d'assistance, permettre de faire face aux pénuries d'héroïne sur le marché noir ou de poursuivre une toxicomanie médicamenteuse moins onéreuse. Le soulagement des tensions émotionnelles, la «pression sociale » (ex : famille, alternative à l'incarcération), la dégradation de l'état de santé ou la survenue d'événements de vie (grossesse, hospitalisation, prison, décès d'un proche, etc.) (Koester et al., 1999; Neale, 1999b; Beschner et Walters, 1985; Hunt et al., 1985) sont d'autres raisons précipitant parfois la demande de traitement. Malgré les bénéfices réels rapportés (Gayre et Richard, 2000), les attitudes et les perceptions à l'égard de la méthadone se révèlent souvent beaucoup plus mitigés du fait de la pénibilité de ses nombreux effets secondaires et son fort potentiel addictif (Beschner et Walters, 1985; Hunt et al., 1985; Rosenbaum et Murphy, 1981; Brown et al., 1975; Koester et al., 1999; Gold et al., 1988; Rosenblum et al., 1991). D'autres travaux mettent directement en cause les pharmacothérapies de substitution pour leur potentiel d'escalade dans les processus de la dépendance vers des formes plus sévères de polytoxicomanies. Ils rendent compte aussi d'un déplacement de la soumission aux règles de la rue à la dépendance à l'institution médicale (Bourgois, 2000; Newman, 1996; Fischer et al., 2002a; Magura et Rosenblum, 2001). Enfin et surtout, ressort la difficulté des usagers à se soumettre au cadre de prise en charge, souvent très strict, de la méthadone (supervision de l'administration des traitements contraints à des horaires délimités, contrôles 
urinaires) et dont les modalités de suivi des traitement sont perçues comme infantilisantes, moralisatrices et brimant la liberté individuelle.

En France, les modalités de traitement proposées aux personnes dépendantes des opiacés se démarquent de ce qui se fait quasiment partout ailleurs. La méthadone n'occupe qu'une place très secondaire dans le dispositif de soin, la buprénorphine haut dosage ${ }^{1}$ ayant été adoptée comme traitement de première intention $(85000$ personnes traitées pour environ 15000 sous méthadone $)^{2}$. À la différence de la méthadone encore largement cantonnée aux centres de soins spécialisés aux toxicomanes (CSST), le Subutex® (nom commercial de la buprénorphine haut dosage) domine la pratique en médecine générale avec des modalités réglementaires de suivi des traitements et de prescription extrêmement souples ${ }^{3}$.

La rapide et très large diffusion du Subutex ${ }^{\circledR}$ a suscité de la part des professionnels de la toxicomanie un discours très critique mettant en cause la valeur de cette stratégie sanitaire et renouant avec le discrédit porté de longue date sur les traitements de substitution comme instrument de contrôle social. Ce discours a été nourri par l'observation de pratiques de consommation intégrées aux usages de drogues de rue (injection, mélange de produits, etc.) et soutient la contestation de cette stratégie de santé publique. Ces usages des produits de substitution ne peuvent être caractérisés par les grilles de la recherche clinique ou de l'observation épidémiologique qui évaluent la conformité des consommations aux normes médicales.

\footnotetext{
${ }^{1}$ La buprénorphine est un morphinique agoniste partiel/antagoniste. Mis sur le marché en 1984 comme antalgique sous le nom de Temgésic ${ }^{\circledR}$, il a pendant longtemps été utilisé par les médecins généralistes, à la limite de la légalité, pour venir en aide aux usagers en l'absence d'autres substances adaptées (Inserm, 1998). En 1996, les autorités sanitaires ont décidé de commercialiser une formulation haut dosage sous le nom de Subutex® pour les traitements de substitution aux opiacés. En France, le Subutex ${ }^{\circledR}$ est disponible sous une forme comprimé. L'injection est alors possible, les excipients non solubles entraînant des complications locales plus ou moins graves. Une prise journalière et une administration par voie sublinguale sont recommandées pour une efficacité pharmacologique optimale (Inserm, 1998).

${ }^{2}$ Source : Données pour l'année 2003 fournies par Julien Emmanuelli - Institut national de veille sanitaire (InVS) SIAMOIS.

${ }^{3}$ La durée de prescription peut s'étendre jusqu'à 28 jours, la délivrance se fait en officine et sans supervision de l'administration, les contrôles urinaires ne sont pas prévus et il n'y a pas d'enregistrement médico-administratif des personnes auxquelles le traitement est prescrit contrairement à d'autres pays. Pour plus d'informations sur le dispositif français des traitements de substitution et le descriptif détaillé des aspects pharmacologiques propres au Subutex ${ }^{\circledR}$, le lecteur est invité à se référer au rapport annuel publié par l'OFDT (O.F.D.T., 2003b)
} 
Les travaux menés auprès des usagers sous méthadone dans des pays utilisant quasiexclusivement ce produit de substitution pour l'indication de dépendance aux opiacés ne suffisent pas à éclairer la situation française marquée par un régime doublement particulier du fait de la place accordée aux médecins généralistes et du choix du Subutex® qui contrastent avec la démarche de « contrôle » de la méthadone dans ces pays. C'est pourquoi une approche qualitative empruntant à la démarche sociologique a été adoptée.

Dans un domaine où la perspective des usagers fait simultanément référence aux propriétés du produit, à ses effets, à son régime thérapeutique, à la scène locale de la drogue et à la singularité des trajectoires individuelles, on s'interroge ici sur le rapport des usagers au Subutex ${ }^{\circledR}$ dans un contexte réglementaire favorisant une relative autonomie dans la gestion de la prescription. En s'appuyant sur une approche globale et qualitative des trajectoires d'usagers dans leur rapport à la toxicomanie, l'étude s'intéresse à la manière dont ce produit s'inscrit dans le quotidien des usagers et à l'évolution du vécu de la toxicomanie au cours de la prescription. Elle a pour objectif d'explorer les différentes façons dont le Subutex® prescrit vient s'intégrer dans les parcours des usagers avec les produits psychoactifs licites et illicites en tenant compte des contextes de vie et des profils individuels des usagers concernés.

\section{METHODES}

L'analyse présentée dans cet article porte sur des entretiens réalisés en 1999-2000 auprès de 28 usagers sélectionnés à partir d'un échantillon plus large de 66 usagers rencontrés dans le cadre d'une étude visant à décrire et à analyser les usages du Subutex® obtenu avec ou sans prescription. Etant donné le caractère exploratoire de l'étude, la stratégie de réduction de l'échantillon final a consisté à ne retenir que les usagers ayant obtenu le Subutex® par une prescription médicale et ayant pu s'exprimer sur toutes les dimensions à l'étude. 


\subsection{Procédure d'échantillonnage et de recrutement}

La stratégie de recrutement s'est attachée à contraster les registres de consommation, les caractéristiques individuelles (genre, âge, vie de couple, emploi), les contextes urbains (Paris et proche banlieue nord-est). Les relais mobilisés ont été les centres de soins spécialisés aux toxicomanes (CSST), les médecins prescripteurs en cabinets libéraux (CL) et les programmes d'échange de seringues (PES). Les critères d'éligibilité adoptés étaient les suivants : avoir plus de 18 ans, avoir consommé plus de deux fois du Subutex ${ }^{\circledR}$ dans le cadre d'un traitement de substitution pour une dépendance à l'héroïne au cours du mois précédant la première entrevue. En milieu médical, il était demandé aux soignants impliqués dans le recrutement de diversifier les usagers eu égard à la régularité de leur suivi (respect des rendez-vous, continuité dans le traitement, demandes de dépannages ou d'avance de la prescription, interruptions du traitement) de façon à rendre compte de l'hétérogénéité des conduites face au traitement. Dans les PES, le seul critère retenu était l'obtention du Subutex® sur prescription médicale. Les usagers sous l'effet marqué de drogue lors de la prise de contact et ceux présentant des comportements ou tenant des propos susceptibles de rendre difficile la conduite des entretiens ont été exclus.

Tous les entretiens ont été menés par le chercheur et auteur principal de cet article, alors en cours de doctorat de santé publique. Durant la phase de recrutement, les usagers ont été systématiquement informés : a) du strict anonymat et de la confidentialité des entrevues, notamment par rapport aux structures de soins b) des modalités et du déroulement de l'enquête, notamment du caractère volontaire, de la série de trois entretiens et de leur enregistrement, c) de la possibilité de refuser les entretiens ultérieurs sans conséquences pour le traitement et sans avoir à se justifier, et d) de l'absence de rémunération. Les modalités de prise de contact ultérieure les plus adéquates pour la personne étaient alors discutées ; le téléphone portable fut l'outil le plus souvent choisi par les usagers pour être contacté de façon à la fois anonyme et personnalisée 


\subsection{Recueil des données}

Un guide d'entretien a été mis au point à partir d'une dizaine d'entrevues exploratoires et des observations menées dans deux structures de soins. Il a permis d'aborder les usages du Subutex ${ }^{\circledR}$ ainsi que les contextes de consommation, le mode de vie des usagers et leurs réseaux sociaux, ainsi que leur état de santé. La méthodologie utilisée s'est inspirée de l'approche phénoménologique des entretiens approfondis (in-depth) décrits par Seidman (1998) et qui adopte une technique d'entretiens semi-directifs répétés dans le temps pour «sonder » l'expérience et la mettre en contexte. Compte tenu du sujet de la recherche qui explorait les différents registres de consommation du produit de substitution, cette méthode a également été retenue pour permettre l'établissement d'un lien de confiance dans le temps et faciliter l'expression. En outre, la répétition des entretiens avait pour objectif de réduire l'effet de discours rencontré souvent lors des premiers contacts avec un usager, de mettre les informations sur l'usage de la substitution en lien avec des éléments concrets de l'histoire de vie, de rendre compte de l'instabilité des modes de vie et des rapports aux drogues et au produit de substitution. De plus, la répétition des entretiens permettait d'obtenir suffisamment d'informations pour des individus qui n'auraient pas supporté des entretiens trop longs. Ainsi, trois entretiens (d'une durée variant de 40 minutes à deux heures) ont été réalisés, les deux premiers rapprochés d'environ 15 jours puis le troisième, trois mois plus tard.

Le premier entretien était centré sur a) le contexte et les conditions d'initiation du Subutex ${ }^{\circledR}$ (histoire avec les produits, traitements antérieurs, éléments d'histoire et de mode de vie, et b) les modalités de la prescription et l'utilisation du Subutex® (dose prescrite, dose consommée, rythme des prises, mode d'administration, autres produits consommés, etc). Le second entretien portait sur la mise en relation par le répondant de ses usages du Subutex® avec son mode de vie (logement, insertion professionnelle, situation juridique/administrative, vie familiale ou affective, etc.) et se basait sur le récit d'une journée (la veille de l'entretien) et le récit des événements survenus depuis la première rencontre. Le dernier entretien répétait cette 
structure et proposait à la personne de faire une synthèse du sens de son expérience avec le Subutex®. Chaque entretien était préparé sur la base des notes consignées dans un journal de bord. Les entretiens ont été réalisés en dehors des structures de soins dans des lieux laissés au choix de la personne : parcs, cafés, squats et plus rarement à domicile. Tous les entretiens ont été enregistrés et intégralement retranscrits.

Sur les 28 usagers rencontrés, la majorité $(n=16)$ est issue de la banlieue proche de Paris. Ce groupe est âgé de 23 à 43 ans et composé principalement d'hommes (n=19). La moitié de l'échantillon a un emploi rémunéré et vit dans un logement stable. Le suivi du traitement est réalisé en majorité dans les cabinets libéraux $(\mathrm{n}=17)$ et pour la quasi-totalité de l'échantillon (sauf 4), la durée de la prescription du Subutex® est supérieure à 2 ans. Au moment des entretiens, deux personnes étaient inscrites dans un suivi psychologique. À l'exception de trois usagers rapportant une toxicomanie récente, tous les autres présentent une longue histoire avec l'héroïne, pour la plupart par injection (24/28), variant de 5 à 10 ans pour 12 d'entre eux, supérieure à 15 ans pour une minorité $(\mathrm{n}=5)$, et consommée presque exclusivement. Au moment de l'enquête, la quasi-totalité des répondants a déclaré l'abandon définitif de l'héroïne et plus de la moitié avoir cessé de s'injecter.

\subsection{Etapes de l'analyse}

Le travail d'analyse a porté sur 84 entretiens, facilité par le recours au logiciel Atlas.ti (version WIN 4.2) dont l'une des caractéristiques est de permettre une gestion simplifiée d'un très grand nombre de données discursives. Outre cette modalité d'usage, Atlas.ti nous a permis de développer les dimensions de la typologie du rapport des usagers au produit de substitution suivant la démarche de la grounded theory pour laquelle le logiciel a été développé. L'élaboration des concepts et de leur dimension repose sur l'analyse textuelle menée à partir d'une codification du matériel. La première étape d'analyse a consisté en une lecture systématique des retranscriptions des notes et des entretiens afin de classer les informations qu'elles contenaient en 
différentes catégories d'analyse (codification ouverte). Certains codes découlaient aussi de l'examen de la littérature, c'est-à-dire qu'ils ont été créés avant la récolte des données et, par conséquent, définis d'emblée comme des concepts (codes induits). Pour la majorité cependant, c'est d'une part l'expérience du terrain et, d'autre part le travail sur les retranscriptions qui les ont suscités (codes déduits). Des étapes successives de codifications ont été effectuées en procédant par itération pour formuler et affiner progressivement les hypothèses sur les relations entre catégories et entre les catégories et leurs propriétés (codification axiale) et faire ressortir les logiques discursives. Ce faisant, nous avons pu mettre en évidence les variations des situations des usagers en traitement au fil de la répétition des entretiens et étudier les conditions et les contextes des phénomènes observés. La dernière étape de l'analyse a consisté à dégager la typologie des profils illustrant le rapport des interviewés au produit de substitution. 


\section{LE RAPPORT DES USAGERS AU SUBUTEX® : CARACTERISATION ET PROFILS}

Les analyses ont permis de dégager cinq axes caractérisant le rapport des usagers au produit de substitution qui renvoient 1) au mode d'appropriation du Subutex $\left.{ }^{\circledR}, 2\right)$ aux usages du Subutex ${ }^{\circledR}, 3$ ) au vécu de la toxicomanie, 4) aux situations sociales, aux réseaux sociaux et aux modes de vie, et 5) et à l'image de soi. Le positionnement des individus par rapport à ces différents axes configure quatre profils dominants : des ajustements tournés vers la réinsertion (profil 1 intitulé « une ressource pour s'en sortir »), une gestion contrôlée des produits (profil 2 intitulé « un traitement de maintenance »), la poursuite et l'aggravation d'une toxicomanie aux opiacés (profil 3 intitulé « une drogue légale»), et une transition vers des abus ou une dépendance plus sévère (profil 4 intitulé « un produit piège »).

\subsection{Caractérisation des profils}

\subsubsection{Mode d'appropriation du Subutex ${ }^{\circledR}$}

Cet axe renvoie à la façon dont les usagers perçoivent et situent le Subutex® dans leur parcours avec les substances (alcool, drogues illicites, médicaments) et dans leurs expériences antérieures avec d'autres produits de substitution (méthadone, Temgésic $®$, sulfate de morphine) qu'ils aient été obtenus dans la rue ou avec une prescription médicale. Le Subutex® est situé dans l'univers des produits expérimentés par l'usager selon les fonctions qu'il remplit et les effets recherchés et ressentis.

L'appropriation du Subutex® se décline selon quatre positions :

a) Un médicament: une ressource transitoire conçue pour mener à une «sortie de la toxicomanie "),

b) Un traitement de maintenance et de confort : un soutien chimique nécessaire pour fonctionner au quotidien, participant d'une meilleure qualité de vie, et dont l'usage est envisagé dans le très long terme, 
c) Une drogue : une substance qui met en jeu une logique d'effets similaires à celle de l'usage de drogues comme l'héroïne ou la cocaïne avec la recherche d'un état de « défonce »,

d) Un produit « diabolique »: une substance devenue instrument de persécution, perçue comme pire que l'héroïne du fait d'une augmentation continue des doses absorbées et de la perception de risques et de dommages aggravés.

\subsubsection{Les usages du Subutex@)}

Dans l'étude, les usages correspondent à la façon dont la personne met en acte, conçoit et donne sens à sa pratique dans sa logique d'acteur (Trostle, 1997). Les usages sont repérés à travers les modes et les pratiques de consommation du Subutex ${ }^{\circledR}$ d'une part, des médicaments prescrits et des autres produits licites et illicites utilisés d'autre part. Ils se caractérisent en fonction de la quantité consommée (sur/sous consommations par rapport à la dose prescrite), du rythme quotidien des prises pour le Subutex ${ }^{\circledR}$ (uniques, fractionnées ou anarchiques); de la consommation d'autres substances (licites ou illicites), du mode d'administration (oral, sublingual, fumé, injecté, sniffé) et de la fréquence de ces pratiques (occasionnelles, régulières, systématiques), et du contexte des consommations (le soir, le matin, le week end, ou en fête uniquement, tout au long de la journée, ponctuellement lors de la survenue d'un évènement stressant, seul ou entre amis....), et des effets recherchés.

Quatre positions ont été dégagées :

a) Le régime individualisé: correspond à l'aménagement de la prescription de Subutex® (prise unique ou fractionnée du Subutex ${ }^{\circledR}$ sur et sous consommation de la dose prescrite) par l'usager pour l'adapter à son «métabolisme» ou à son mode de vie. Une fois défini, ce régime devient la «norme thérapeutique » de l'usager qui s'y conforme dans le temps. Le Subutex® est ici consommé principalement par la voie sublinguale et s'accompagne d'usages occasionnels et festifs de cocaïne et d'héroïne, 
b) L'observance : renvoie à la conformité de l'usager à la prescription et aux consignes médicales relatives au Subutex ${ }^{\circledR}$ (dose, mode d'administration....). Dans ce positionnement, on ne relève pas de consommations de cocaïne, d'héroïne ou de crack,

c) L'intégration du Subutex $($ aux drogues de rue : correspond à l'intégration du produit de substitution aux anciens systèmes de consommation avec un recours dominant à l'injection ou au sniff, l'usage associé ou en alternance de différentes substances licites, illicites - alcool benzodiazépines- ou illicites -héroïne, cocaïne, crack-),

d) Les usages chaotiques du Subutex®: se caractérisent par des modes et des pratiques de consommation aléatoires du Subutex ${ }^{\circledR}$ et des autres substances légales, illicites ou détournées disponibles dans la rue.

\subsubsection{Le vécu de la toxicomanie}

Cet axe correspond aux signes ressentis de la dépendance avec le Subutex®, aussi bien physiques (manque et « $\left.\mathrm{craving}^{4} »\right)$, psychologiques (obsession), que comportementaux (compulsion). Quatre catégories composent cet axe avec en toile de fond une dépendance physique recouvrant le sentiment du déplacement de la dépendance au dealer à l'institution médicale :

a) La régulation-stabilisation du rapport au(x) produit(s), y compris le médicament de substitution correspond au sentiment de reprise de contrôle et de maîtrise de ses consommations,

b) La substitution et la maintenance renvoient à l'intégration par les usagers des principes d'un médicament qui soulage durablement le manque et atténue l'envie de consommer,

c) La chronicisation correspond au sentiment d'une dépendance à l'héroïne (ou aux produits en général, selon les parcours) renforcée par le Subutex®: pas d'évolution perçue du rapport au(x) produit(x) consommé(s) antérieurement et forte assuétude,

\footnotetext{
${ }^{4}$ la notion anglophone de « craving » revoie au désir obsédant et irrépressible de prendre un opiacé, et donc à l'incapacité de maîtriser sa consommation
} 
d) La dépendance «totalisante », associe les sentiments de perte de contrôle aggravée de la consommation de produits et de forte emprise de l'institution médicale et plus largement des pouvoirs publics. Cette notion fait également référence à l'imposition d'une sorte de discipline du comportement par le produit qui se traduit par la survenue d'effets secondaires indésirables sanctionnant les modes de consommation du Subutex® non-conformes à l'indication thérapeutique (sniffé, le Subutex ${ }^{\circledR}$ ne procure qu'un plaisir modeste; injecté, il produit une «défonce » médiocre pour des risques d'abcès sévères; la prise de Subutex ${ }^{\circledR}$ consécutive à l'héroïne inflige une crise violente de manque).

\subsubsection{Modes de vie et réseaux sociaux}

Le toxicomane est selon Robert Castel (1998) celui qui organise toute son existence autour de la recherche et de la consommation de drogue, ou encore comme l'écrit Albert Ogien (1995) celui qui fait de la drogue la seule ligne biographique de son existence. De leur point de vue, la consommation de drogues (achat et consommations) est devenue une préoccupation constante qui domine la vie sociale et structure le mode de vie dans les activités du quotidien. Le consommateur intégré réussit, à l'inverse, à faire cohabiter différentes lignes biographiques (travail, famille) avec sa consommation de drogue.

Cet axe du mode de vie est donc très présent dans la façon dont les usagers expriment leur rapport au produit de substitution. Compte tenu de la multiplicité des facteurs qui entrent dans la situation sociale, on distingue ici moins des catégories que des pôles : d'un coté la normalisation du mode de vie, de l'autre le maintien d'un style de vie marqué par la sous-culture de la vie à la rue. Certains parviennent à des degrés divers à investir de nouveaux centres d'intérêts et à se dégager de l'emprise du milieu de la rue et de la drogue. Cette normalisation s'appuie sur des aspects relationnels (ouverture vers les autres, élargissement du réseau social hors de la sphère des produits, nouveaux investissements affectifs...), occupationnels (diversification des centres 
d'intérêts et des loisirs, activités physiques et sportives), les activités professionnelles et familiales (adoption de nouveaux rôles sociaux: vie de couple, projet d'enfant, activités militantes, participation associative, etc). À l'autre extrême se positionnent ceux pour qui la consommation de drogue reste une préoccupation dominant la vie sociale et qui conjuguent ou cumulent l'absence de logement fixe, d'emploi, l'isolement, l'imprévisibilité du quotidien et des relations sociales tournées quasi-exclusivement vers des usagers. On nommera cette position : marginalisation.

\subsubsection{L'image de soi}

L’image de soi se dégage comme une composante essentielle au sein de la problématique identitaire qui se joue dans le décours de l'expérience du Subutex®. Elle apparaît de façon forte dans le discours des usagers sur leur rapport à la consommation de drogue et au Subutex®. Elle correspond à la façon dont l'usager se perçoit en référence à la figure réprouvée du «junkie » dans le regard, les propos et les attitudes de l'entourage. L'analyse des entretiens dégage quatre catégories dont certaines ont déjà été identifiées dans différents travaux : une image d'ancien toxicomane (« ex-tox») dans laquelle le stigmate est en quelque sorte atténué, celle du toxicomane invétéré (" always a junkie»), celle de malade (patient), enfin celle de « cobaye », correspondant à l'impression de faire l'objet d'une expérience «socio-médicale » aux intentions douteuses.

\subsection{Présentation des profils}

Quatre profils se dégagent de la combinaison de ces cinq axes pour rendre compte des rapports différenciés des usagers au Subutex® (tableau 1). Chaque profil est illustré par un cas présenté de façon détaillée dans un encadré.

\section{Insérer Tableau 1}




\subsubsection{Profil 1 : Une ressource pour s'en sortir:}

Yves, 35 ans est aide soignant, sa conjointe est infirmière. Le Subutex ${ }^{\circledR}$ " remet les yeux en face des trous", dit-il. Il apporte un soulagement mais amène aussi une prise de conscience brutale et violente de tout ce qui a été occulté par l'hérö̈ne. Il se sent démuni pour faire face à la réalité sans l'" assurance" que procurait l'héroüne. Le soutien et la confiance de ses amis et de sa femme l'encouragent. Son travail l'occupe, lui offre une base sécurisante pour l'avenir. Yves s'est investi dans la vie du foyer et de nouvelles activités. Il vient aussi d'acquérir un pavillon grâce aux économies réalisées sur l'hérö̈ne. Enfin, se considérant "rescapé " du VIH et de l'hépatite $C$, il tient à " honorer ce cadeau de la vie ». Il affirme vouloir " rattraper le temps perdu » en prenant des responsabilités et en se rendant disponible pour les autres. Sa paternité le réjouit autant qu'elle le confronte. Il est fier de ses nouvelles fonctions de leader syndical. Le Subutex ${ }^{\circledR}$ produit un engrenage qui le tire vers le haut. Pourtant il déclare : " plus je $m$ 'en sors et plus j'ai peur de tout perdre du jour au lendemain et de tomber d'autant plus haut ».

Ce mieux tient selon lui à une gestion très stricte du secret de son traitement. Après une période d'approvisionnement parallèle, il a depuis un an recours à un médecin pour rompre avec le milieu et les valeurs de la rue. Il fractionne la dose en trois prises par jour pour éviter la sensation de défonce quand il prend ses $10 \mathrm{mg}$ d'un coup. Il lui arrive d'augmenter la dose quand il se sent fragilisé. Il revient cependant rapidement à la dose prescrite. Il n'a plus consommé d'héroüne depuis un an mais prend parfois de la cocaïne. Après une consommation de défonce associant Subutex ${ }^{\circledR}$ et alcool, puis alternant héroïne et Subutex ${ }^{\circledR}$, il s'est stabilisé.

Cette stabilisation se traduit aussi, selon ses dires, par le passage du Subutex ${ }^{\circledR}$ en sniff à une prise qu'il qualifie de "normale " sous la langue ; cette normalisation est mise en relation avec la prochaine naissance de son enfant.

Il souffre de l'image du toxicomane qui lui colle à la peau. Il s'est déclaré sous un nom d'emprunt au médecin pour éviter tout étiquetage et ne recourt pas au remboursement craignant de perdre toute sa crédibilité si son traitement était mis à jour dans son entourage, professionnel surtout. Il est déterminant pour lui de se libérer du Subutex ${ }^{\circledR}$ au plus vite.

Un médicament pour sortir de la dépendance : les effets normalisants du Subutex ${ }^{\circledR}$

Le Subutex® est perçu comme un «médicament» quand il fait franchir une étape significative dans la redéfinition de soi et de son rapport avec les substances. Ce sont les qualités d'un produit aux effets «normalisants » qui sont soulignées : un état de bien-être physique sans les sensations de manque ou de défonce. Le passage d'une logique de "défonce » à la seule gestion du manque d'une part, l'abandon de l'injection ou du sniff pour la voie sublinguale d'autre part, évoquent chez tous une sorte de normalisation du rapport au produit. Ce changement est vécu comme une étape décisive dans l'expérience du produit, faisant passer d'une logique de recherche «d'effets drogue » à une logique thérapeutique. L'autre fonction «normalisante »du Subutex ${ }^{\circledR}$ est celle de révélateur des dimensions occultées par la prise de drogue : la satisfaction 
de renouer avec des sensations, des émotions et une façon d'être plus authentiques est aussi une épreuve de lucidité, jugée incontournable pour s'en sortir. La survenue d'épisodes « dépressifs » consécutifs à cette pensée réflexive est récurrente dans les discours.

Le Subutex ${ }^{\circledR}$ s’inscrit dans un projet de « sortie de la dépendance » quand il commence à « sortir de la tête », c'est-à-dire lorsqu'on oublie à plusieurs reprises de le prendre. D'après les répondants, ce n'est qu'une fois dégagé des aspects obsessionnels et compulsifs de la toxicomanie (dont les angoisses liées à l'anticipation du manque) qu'une véritable démarche pour réduire progressivement la dose jusqu'au sevrage peut être amorcée.

\section{Des régimes individualisés}

Les usages du Subutex ${ }^{\circledR}$ se justifient ici dans leur fonction d'intégration, en référence à un ensemble de valeurs socialement reconnues : le soin de soi, le bien-être, le divertissement, la performance ou encore la convivialité. Les usages ne répondent pas dès lors à une simple pulsion de manque mais ils s'inscrivent dans un cadre précis. Ils se distinguent par des modalités de gestion des quantités, des effets et des circonstances de consommation qui visent à ne pas interférer avec les activités du quotidien et à anticiper les conséquences négatives : le médicament est pris le plus souvent en une seule fois, parfois en prises fractionnées, sous la langue et à la dose prescrite. La relation dose/effet est primordiale pour les usagers, d'où le jeu sur le nombre de prises et les doses. Quand il existe, le fractionnement vise à limiter la sensation de défonce. Il s'agit donc de trouver l'équilibre subtil permettant à la fois d'utiliser pleinement ses capacités sans ressentir le manque ni la sensation d'être « défoncé », tout en résistant à l'escalade.

Utilisés plutôt en soirée, le cannabis et les benzodiazépines (obtenues sur prescription) sont décrits comme le pendant psychologique du Subutex ${ }^{\circledR}$, véritables régulateurs des tensions émotionnelles et du sommeil. Les produits illicites (cocaïne, héroïne ou crack) sont peu recherchés et leur usage reste occasionnel (moins d'une fois par mois) restreint à des contextes festifs en compagnie d'autres consommateurs s'inscrivant dans la même logique. 
Normalisation du mode de vie et émancipation du milieu de la drogue

Parce qu'il évite le manque, le Subutex ${ }^{\circledR}$ offre de rompre avec un mode de vie hyperactif centré sur la recherche d'argent et d'héroïne. Sa disponibilité sur prescription favorise la prise d'autonomie par rapport au milieu des drogues (pairs consommateurs et dealers), la réduction des risques d'interpellation et de rechute, et l'ancrage dans un système de valeurs plus commun. Chez les personnes dont les situations sociales, professionnelles et affectives offrent le plus de ressources et de soutien, le Subutex ${ }^{\circledR}$ sert aussi de révélateur des atouts pour s'en sortir. Il offre des perspectives positives pour l'avenir. Les discours illustrent l'émergence ou le retour de centres d'intérêt alternatifs aux produits, tournés vers soi (s'occuper de sa santé, de son corps, de son alimentation, de son aspect vestimentaire), vers d'autres activités, solitaires ou partagées (travail, sport, cinéma, lecture), ou encore vers des satisfactions plus relationnelles (familiales surtout). L'implication et l'engagement dans de nouveaux rôles sociaux (vie de couple, parentalité, accès à la propriété, responsabilités professionnelles) caractérisent les parcours avec le Subutex ${ }^{\circledR}$ comme des éléments qui structurent une identité sociale positive (valorisation, estime de soi...) et se combinent en «boucles de résilience » (élargissement et diversification des réseaux de sociabilités).

\section{«Ex-tox » : le stigmate du Subutex ${ }^{\circledR}$}

Dans la majorité des cas, la prise d'un produit légal n'a pas permis de s'extraire de l'image sociale du «junkie » auprès de l'entourage (famille, amis, collègues) même si pour l'usager les traits qui caractérisent le « junkie » n'appartiennent plus à son quotidien. Le maintien d'une dépendance physique importante et le stigmate liés à la prise du Subutex ${ }^{\circledR}$ sont perçus comme un déplacement des problématiques de la déviance et de la dépendance vers l'institution médicale et une représentation de soi comme « ex-tox ». La dépendance persistante, doublée de la nécessité de maintenir le secret du traitement, menace les acquis et renouvelle l'obsession du sevrage. 


\subsubsection{Profil 2 : un traitement de maintenance}

Victor a 37 ans et vit seul. Divorcé, il a la garde de sa fille de 8 ans un week-end sur deux. Il est en invalidité depuis 2 ans après une dépression liée au traitement de son hépatite C. Pendant des mois, il a cherché et fini par obtenir un stage de reconversion en informatique.

A 28 ans, après cinq années d'héroüne prise en injection, Victor a épousé sa partenaire enceinte. Il a alors arrêté complètement l'héroüne pour une "défonce acceptable " alliant alcool, codéine et benzodiazépines. Il a alors obtenu du Temgésic. Au bout de deux ans, il doit entrer en cure de désintoxication pour l'alcool. Il abandonne alors alcool et tabac mais poursuit le Temgésic qu'il s'injecte moins systématiquement. A cette époque, le Subutex ${ }^{\circledR}$ est mis sur le marché. Il rompt alors avec la seringue, changement qui fait passer le produit dans le registre du médicament. Il est maintenant sous Subutex ${ }^{\circledR}$ depuis 4 ans. Il se qualifie de "bon patient" : il ne prend rien d'autre que son traitement prescrit d'antidépresseur. Il attribue deux fonctions principales au Subutex ${ }^{\circledR}$ : traiter le manque et prévenir les rechutes. "Le Subutex ${ }^{\circledR}$ est à l'héroïne ce que l'Espéral est à l'alcool», dit-il. Il veut "être bien sans être mal ». Considérant la toxicomanie comme une maladie, il situe le Subutex ${ }^{\circledR}$ dans le registre du vital. C'est un traitement comme un autre qu'il rapproche de celui du diabète ou l'hypertension. Il ne se sent plus "toxicomane " et ne supporte pas qu'on lui dise qu'il est "substitué ». Dans sa famille, on fait l'amalgame substitution/toxicomanie. Il dit donc qu'il est "sevré " pour faciliter la confiance et la restauration des liens familiaux. Dans une existence où tout lui semble fragile et incertain, Victor ne conçoit pas l'arrêt du Subutex ${ }^{\circledR}$ (même à long terme), par crainte de rechute. Il le prend indistinctement du reste de ses médicaments en 3 prises régulières afin précise-t-il "de me défaire de tout ce qui est compulsif dans mon comportement ". Il sent avoir repris de contrôle sur le produit. Il ressent un plus grand confort sur le plan sexuel. Il thésaurise la petite économie qu'il fait sur la prescription comme symbole jour après jour sa victoire sur les produits. Il redoute le retrait du Subutex ${ }^{\circledR}$ du marché. Il ne dépanne plus les amis qui en étaient arrivés à le considérer comme un dealer. "Les drogues, dit Victor c'est tellement loin tout ça pour moi, aujourd'hui c'est pour ma santé que j'ai peur, c'est ma tête et l'éducation de ma fille qui me préoccupent et puis j'aimerais bien rencontrer quelqu'un surtout ». La psychothérapie qu'il suit depuis deux ans occupe une grande place dans sa vie. La solitude est pesante depuis qu'il a rompu avec le milieu de la rue. Il s'est impliqué dans le syndicat de son immeuble, va quotidiennement à la bibliothèque avant d'aller chercher sa fille à l'école. Au fil du temps, l'inactivité professionnelle et la solitude alimentent le découragement et l'amertume face à tous les efforts consentis pour s'en sortir.

Entre traitement de maintenance et médicament de confort

Le médicament de substitution assure une forme de survie et participe à une meilleure qualité de vie. Il a permis de rompre la course aux produits de rue. Il relève à la fois du registre du besoin quand il est nécessaire pour démarrer la journée et «assurer » les activités du quotidien, et du registre du confort quand on y puise des effets conjuguant bien-être physique et émotionnel. Dans ce cas de figure, c'est la pénibilité des conditions (ou du maintien) de l'insertion, une santé dégradée, et l'épuisement des années de toxicomanie qui ressort. Les 
existences sont marquées par la souffrance et la vulnérabilité psychologique (états dépressifs), physique (maladie) et sociale (chômage de longue durée, isolement). Le Subutex ${ }^{\circledR}$ remplit parfois le rôle d'adjuvant thérapeutique aux traitements des maladies graves (VIH, VHC), procurant un certain «confort psychologique » pour les uns, atténuant les douleurs physiques pour les autres.

Dépendre d'un produit est ici conçu comme une maladie que certains n'hésitent pas à situer dans le registre d'une «maladie chronique » (au même titre que le diabète) et qui envahit l'ensemble de l'expérience de la personne. Son traitement reste cependant quelque peu à part. L'objet de la substitution navigue entre le soin de « la maladie des produits » (le Subutex® étant au toxicomane ce que l'insuline est aux diabétiques), le soutien à des « polyhandicaps » de la vie, et le traitement palliatif. Le produit de substitution offre un relatif confort pour affronter la réalité sans la protection des drogues et fait de la dépendance une préoccupation secondaire, pour certains absente. C'est la fragilité des modes de vie qui ressort, le souci constant de préserver les acquis (le travail pour ceux qui en ont), et de répondre aux impératifs du quotidien (s'occuper des enfants).

Observance de la prescription et intégration du Subutex ${ }^{\circledR}$ dans les modes de vie

L'observance des recommandations médicales domine ici. Tous consomment le Subutex® sous la langue et à la dose prescrite avec parfois un aménagement en 2 ou 3 prises réparties dans la journée. L'usage du Subutex $®$ tend à s'intégrer aux « rituels » matinaux (avec le café, après la douche, etc...) ou à se combiner aux autres traitements (tri-thérapie anti-VIH souvent mentionnée). Certains ont rapporté une prescription de benzodiazépines, mais la consommation de cannabis ou de produits illicites a disparu depuis plusieurs années.

La maintenance dans la dépendance 
Le Subutex ${ }^{\circledR}$ s’inscrit dans la continuité de l'expérience de l'héroïne : il ne « défonce » plus et laisse place à un état « normal » pour pouvoir fonctionner. Dans ce groupe, les produits de rue sont traités comme d'une expérience dépassée («j'ai tellement pris de distance par rapport à ça... », Béatrice, 43 ans) qui ne «prend plus la tête ». Dans ce contexte, les usagers tendent à construire autour de la maintenance de leurs états de dépendance une problématique relevant essentiellement du registre somatique. Il est perçu comme une barrière pharmacologique (un «garde fou ») qui vient suppléer le manque de volonté ou faire rempart aux tentations qui surviennent encore parfois

Une redéfinition de soi dans le « rôle de patient »

L'image de soi tend à se redéfinir dans l'investissement du « rôle du patient» («sick role ») fortement assise sur le diagnostic de pathologies somatiques sévères (hépatite $\mathrm{C}$, infection à VIH, insuffisance cardiaque) à côté de la toxicomanie. Dans cette conception «globalisante de la maladie », la dissociation entre le suivi de la toxicomanie et le suivi des autres problèmes de santé s'atténue. Il n'est donc pas rare que ces usagers confient le suivi somatique au médecin qui leur délivre le médicament de substitution.

\subsubsection{Profil 3 : une drogue légale}

Diane a 33 ans, elle vient d'emménager dans un studio et ne s'y sent pas en sécurité. Redoutant d'être relancée pour "des plans came ", elle ne répond plus au téléphone. Rmiste, elle fait un stage en bureautique.

Depuis l'âge de 16 ans, elle a consommé de l'héroüne, d'abord en vivant plusieurs années avec un dealer qui la fournissait gratuitement. A 30 ans, elle apprend son hépatite $C$, rompt avec son ami et s'éloigne du milieu. Elle se fait prescrire du Di-Antalvic pour décrocher, diminue la fréquence des shoots, puis alterne Di-antalvic et hérö̈ne jusqu'à abandonner complètement héroüne et seringue. Elle réalise alors qu'elle est " accro » au Di-antalvic, une dépendance pire que l'héroüne, selon elle. Dès la mise sur le marché du Subutex $\mathbb{\circledR}$, Diane obtient une prescription initiale de $8 \mathrm{mg}$ et diminue progressivement la dose sur une période d'un an pour se stabiliser à $2 \mathrm{mg}$ /jour. "Le traitement marchait super bien avec moi ", dit-elle. Survient alors le suicide de son frère. Pour ne pas replonger, Diane remonte à $6 \mathrm{mg}$. Et depuis deux ans maintenant, plus rien ne va avec le Subutex ${ }^{\circledR}$ qu'elle consomme comme " un bonbon », dit-elle, quand elle se sent angoissée, contrariée, s'ennuie ou croit sentir le manque arriver tout en étant consciente de l'absurdité de son comportement. "Dépressive " les lendemains d'abus, elle prend du Lexomil 
prescrit par un médecin autre que celui qui la suit en substitution. Chaque mois, elle tombe en panne de Subutex ${ }^{\circledR}$ et c'est la " galère " des dépannages (pharmacies, CSST, coprescriptions, amis..) et des états de manque. Au troisième entretien, Diane révèle une consommation de crack et des difficultés grandissantes avec le Subutex ${ }^{\circledR}$ utilisé pour atténuer les " descentes 》 de crack. Ses problèmes financiers s'aggravent. Elle a envisagé d'aller se procurer le médicament dans la rue, mais hésite à franchir le pas. Elle a abandonné son stage. Isolée, mal à l'aise avec "les gens ordinaires ", elle a repris contact avec d'anciens amis-amants, comme elle sous Subutex ${ }^{\circledR}$, et réengage une relation affective avec l'un d'entre eux. "C'est pas de l'amour, mais ça fait du bien de pouvoir parler à quelqu'un qui connaît tout ça », dit-elle. Elle fait l'expérience humiliante d'être rejetée par un homme qui a découvert fortuitement une boîte de Subutex $\mathbb{R}$. Pour elle, la prise du Subutex ${ }^{\circledR}$ est attachée au même stigmate que celui de la toxicomanie.

Pourtant, le Subutex ${ }^{\circledR}$ reste moins dangereux que l'héroüne, la sécurise par rapport à l'overdose, la tient à l'écart des " cachetonneurs ${ }^{5}$ ». Diane apprécie son caractère " normalisant". Débarrassée de l'injection, coquette, elle peut maintenant montrer ses bras et porter des tenues plus féminines.

\section{Une drogue : gestion du manque et défonce}

Le Subutex® est perçu comme une drogue qui reproduit les effets et le mode d'action des opiacés. Son goût «amer » et les démangeaisons sont typiques de l'héroïne en sniff. A part le « flash », les effets du Subutex ${ }^{\circledR}$ répètent le phénomène en « dent de scie » de l'héroïne, c'est-àdire une sensation de chaleur de quelques minutes qui s'atténue progressivement jusqu'à laisser place à un mal être physique ou psychologique que seule une nouvelle prise soulage. La recherche d'effets intenses est au premier plan. Les expressions pour décrire les effets du Subutex® empruntent en tout point au registre de l'héroïne : «piquer du nez», «montée », «taper» etc. La description de ces états évoquent ceux produits par l'héroïne avec la sensation d'une conscience modifiée et diffuse, un état de bien être hors de soi et protégé des turbulences du monde extérieur. En fonction de leur expérience, les uns voient un retour des effets des opiacés (ceux qui ont arrêté depuis longtemps l'héroïne), alors que pour d'autres, il s'agit d'une substance venue s'intégrer dans une histoire récente (voire initiatique) avec les opiacés et d'autres médicaments détournés. Et à l'instar de l'héroïne, les effets plaisants du Subutex® s'estompent avec le temps.

\footnotetext{
${ }^{5}$ L'expression « cachetonneurs » qualifie les usagers consommant divers médicaments psychotropes en association avec de l'alcool ou du Subutex ${ }^{\circledR}$ avec pour effet recherché la potentialisation de tous les produits consommés.
} 


\section{Intégration du Subutex ${ }^{\circledR}$ aux drogues de rue}

Le Subutex ${ }^{\circledR}$ n'est pas pris oralement, il est largement surconsommé par rapport à la dose prescrite en multiples prises quotidiennes. Chez les moins expérimentés, le Subutex® peut suffire à lui seul à remplir les fonctions du produit-drogue. Parmi les vieux consommateurs d'héroïne, le Subutex ${ }^{\circledR}$ est une drogue parce qu'il offre une «défonce » satisfaisante et économique surtout lorsqu'il est injecté et/ou associé à d'autres substances, en particulier le cannabis, l'alcool et les benzodiazépines. Certains consomment héroïne, cocaïne ou crack ou des amphétamines et des drogues de synthèse. Il s'agit d'usages occasionnels en fonction des opportunités du marché et des rencontres de la rue ou des moyens financiers. Pour d'autres, le Subutex® est utilisé pour adoucir les descentes de speed, pour pallier les pénuries d'héroïne, ou pour réduire les coûts de ces consommations.

\section{Chronicisation de la dépendance par le produit de substitution}

Chez les vieux consommateurs d'opiacés, le Subutex® est intégré dans la continuité des logiques qui préexistaient. Il entretient le désir d'effets forts, une dépendance physique importante et l'anticipation du manque. C'est un nouveau produit dans un système de consommation ancien où l'injection constitue un mode d'administration privilégié ou normal des produits. Parmi les injecteurs, la galénique du Subutex ${ }^{\circledR}$ qui permet l'injection est jugée peu favorable à l'adoption d'un rapport nouveau avec le produit. Le sentiment d'une dépendance à la seringue retient dans une logique drogue avec tous les risques de cette pratique avec le Subutex® (dégradation des veines, abcès). Ceux qui ont tenté de « décrocher » depuis longtemps évoquent le registre plus spécifique d'une chronicisation de la dépendance par le médicament et le sentiment d'une emprise chimique renforcée. Le Subutex ${ }^{\circledR}$ s'insère par déplacement dans la continuité de l'expérience des opiacés et des (auto)substitutions successives : la tolérance s'installant sans procurer de plaisir et punissant de surcroît par ses propriétés pharmacologiques (effet seuil, antagonistes, etc...) les comportements tournés vers la recherche d'un plaisir. Dans 
l'incapacité de réduire les doses, les usagers se sentent, pris dans une logique d'échecs à répétition et c'est le sentiment d'une captivité dans la substitution qui prend le dessus.

\section{Une dépendance à moindres risques}

Chez les consommateurs plus jeunes, qui ont une histoire plus récente, marquée par des consommations multiples de médicaments détournés et de substances multiples, le Subutex® marque l'entrée dans la dépendance aux opiacés (ils n'ont jamais connu d'états de manque avant). La pénibilité de la dépendance au Subutex® est secondaire à la fonction de réduction des risques qu'il sert. Il apparaît plutôt comme une ressource dans l'économie de la dépendance qui contribue à réduire les coûts et les risques de la dépendance à une drogue de rue. Les usagers mettent en avant les bénéfices d'un produit satisfaisant, économique, dont l'accès dans la rue est facile et relativement sécurisé puisqu'il n'oblige pas à passer par le réseau des vendeurs de produits illicites comme l'héroïne, la cocaïne et le crack. Se comparant aux utilisateurs d'héroïne marqués par la dégradation sociale et physique qu'entraîne largement, selon eux, le coût et les modalités d'approvisionnement de la substance, les usagers évoquent le très bon rapport « qualité-prix »du Subutex ${ }^{\circledR}$. Ainsi, « décrocher » est très secondaire dans la hiérarchie des préoccupations.

\section{La marginalisation renouvelée $d u$ « junkie »}

Dans le registre social, la prise de Subutex ${ }^{\circledR}$ ne s'accompagne pas d'une évolution significative des conditions de vie. La plupart demeurent confrontés au quotidien de la rue et du commerce des produits. A leurs yeux, comme à ceux de leurs proches, rien n'a changé : pas d'emploi, pas de logement personnel, mêmes fréquentations, toujours «junkie ». Encore soumis aux systèmes de survie dans la rue, ils continuent de faire la manche et à participer à des activités illicites, mais bien moins activement qu'avant. Pour quelques uns, la revente du Subutex® participe d'une économie de survie menée sans scrupules car sur leur échelle des risques et des 
dommages, ce commerce est sans grand danger : pas de concurrence parmi les revendeurs, des prix de vente du Subutex ${ }^{\circledR}$ fixés avec souplesse sans réels enjeux économiques, un profil «client » plutôt amical dans un contexte de dépannage.

\subsubsection{Profil 4 : un produit piège}

Paulo a 35 ans et vit en squat depuis 3 ans. Il touche le RMI auquel s'ajoutent des revenus tirés d'activités illégales. Une quinzaine d'années de polytoxicomanie (héroüne, cocaïne, amphétamines, médicaments) suivie de plusieurs années d'usages de médicaments de substitution

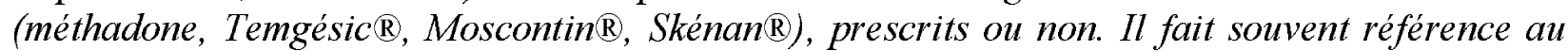
Temgésic $®$, " un produit miraculeux », dit-il. Il lui a permis de franchir un pas significatif, de se refaire une santé, de retrouver un travail, et de renouer avec sa famille, même s'il l'injectait. En 1995, il est incarcéré. A sa sortie, sa femme l'a quitté, plus de logement, le Subutex ${ }^{\circledR}$ remplace le Temgésic $®$. Résolu à ne plus toucher à l'héroüne, il se tourne vers le Subutex ${ }^{\circledR}$. Epuisé, précarisé et en mauvaise santé (hépatite C), Paulo n'est plus du tout attiré par la "défonce ", il recherche un produit qui dynamise, procure un confort psychologique. Ce qu'il trouve en associant Subutex@ et Rohypnol®, parfois des amphétamines ou de la cocä̈ne. Après deux années

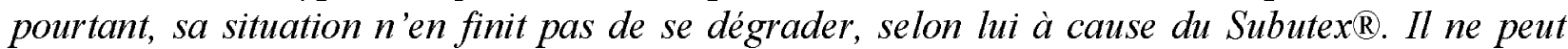
cesser de l'injecter, ses veines sont dans un état alarmant. Pour Paulo, c'est un produit incontrôlable et traître : "comme on le sent pas, on en prend, on en prend et puis tout à coup on se rend compte qu'on est complètement accroc mais c'est trop tard.... ", explique-t-il. Il se sent pris au piège. Il n'arrive pas à prendre le comprimé par la bouche. Il a échoué dans sa tentative de sevrage bien pire, pour lui, que celui d'hérö̈ne. Il en revient au Subutex ${ }^{\circledR}$ " par contrainte et désespoir ", dit-il. Il refuse drogues, méthadone ou sulfate de morphine : "je n'en peux plus, mon corps n'en peut plus ", dit-il. Au fil des difficultés grandissantes à s'injecter, il décrit des états de manque quasi permanents, soulagés par des "cocktails explosifs" (alcool, amphétamines, benzodiazépines), dit-il. Il commet alors des actes dont il ne garde aucun souvenir. Montrant un abcès découvert au réveil, il déclare : "Voilà, c'est juste pour que vous vous rendiez bien compte ce qu'il fait le Subutex ${ }^{\circledR}$ ». Rien n'a changé dans sa vie, Paulo est toujours à la rue. Il a rompu avec le milieu de l'hérö̈ne, et fraye avec les "cachetonneurs ». Il garde de bonnes relations avec sa sæur et sa mère, toujours méfiantes et infantilisantes à son égard. Le Subutex ${ }^{\circledR}$ ne fait pas la différence à leurs yeux. Dans l'univers de la rue, il est avec les autres usagers de Subutex ${ }^{\circledR}$ mis à l'écart.

Au troisième entretien, Paulo se dit au bout du rouleau, contraint d'arrêter le Subutex ${ }^{\circledR}$ car sa vie en dépend. Il livre alors des motivations plus intimes touchant à l'image du corps (traces profondes des abcès à répétition) et à la difficulté à avoir des relations sexuelles satisfaisantes depuis le Subutex ${ }^{\circledR}$, ce qui accentue sa mésestime de lui-même. Il s'isole encore un peu plus. Dans une ultime tentative de reprise de contrôle, il part se sevrer loin de Paris et sans en référer au médecin qui lui prescrit le Subutex ${ }^{\circledR}$ qu'il considère responsable de sa situation.

Un produit diabolique

Les usagers se sentent pris au piège d'un produit «diabolique » qui contraint à une escalade sans limite en l'absence de repères régulateurs ou d'effets modérateurs (absence de 
plaisir ou de satisfaction psychologique, absence de repères corporels et de sécurité par rapport aux risques d'overdoses). Pour les uns, il ne soulage pas le manque induit par des années de polyconsommations intenses, pour les autres, il n'apporte pas le soulagement psychologique attendu. L'état de «manque psychologique» provoque un mal être viscéral décrit comme «un grand vide intérieur », « une boule omniprésente », « quelque chose de cassé à tout jamais », et qui conduit à l'inhibition et au repli sur soi. L'intense détresse psychologique attribuée au Subutex ${ }^{\circledR}$ peut atteindre une dimension existentielle chez ceux marqués par la prison, la vie à la rue, les ruptures affectives et un mauvais état de santé. Il ne permet pas le mieux être même transitoire des drogues de rue et laisse à vif la conscience de la situation et d'un avenir compromis. La facilité d'obtention du Subutex ${ }^{\circledR}$ dans la rue ou sur prescription ne permet plus les pauses dans la consommation auxquelles le coût de l'héroïne ou les pénuries du marché contraignaient. Enfin, la découverte du manque, plus pénible que celui de l'héroïne, constitue un évènement clé de la perception du traitement comme un piège.

\section{Des usages chaotiques}

Le Subutex® est au cœur d'une consommation erratique tendue vers la recherche d'effets forts. Les doses, les rythmes et les modes de consommation (par injection le plus souvent) n'ont plus rien à voir avec les recommandations médicales. Le refus inconditionnel de retourner vers l'héroïne souvent arrêtée de longue date et le manque de moyens poussent à se tourner vers «n’importe quelle » substance, selon les aléas des ressources et du marché de la rue.

\section{L'impasse d'une dépendance totalisante :}

La «dépendance à l'injection » entretenue par la possibilité d'injecter les comprimés contribue fortement à cette impression de piège. Cette possibilité d'injection suscite l'incompréhension, la frustration et le désespoir de ceux qui se perçoivent dans l'incapacité d'arrêter une pratique devenue pénible et dangereuse. Les abcès à répétition et la détérioration 
des veines obligent à recourir à des sites d'injection dangereux (dans le cou) ou à partager des seringues quand les aiguilles se cassent. L'exaspération des tentatives de résister au « craving », les états de manque de plus en plus douloureux s'achèvent dans ce qu'on pourrait appeler « les cocktails du désespoir », avec des quantités massives et désordonnées de médicaments et d'alcool produisant un état d'abrutissement complet. Sous l'emprise des effets désinhibants et/ou anesthésiants de certaines associations (Subutex ${ }^{\circledR}$ et Rohypnol® par exemple), les usagers dressent la liste des comportements et des dommages consécutifs à ces consommations : passages à l'acte incontrôlés, confusion mentale, amnésies et troubles de la mémoire, troubles du sommeil, comas, abcès, etc...).

Dans ce contexte, les répondants évoquent une dépendance ultime et aggravée que l'on pourrait qualifier de totalisante au produit, à la seringue, et à ceux qui sont chargés d'en assurer la délivrance. Elle a envahi le corps, l'esprit, «l'âme », pour aboutir à l'impasse des recours. Arrivés à un point de rupture avec le Subutex ${ }^{\circledR}$, enchaînés à un produit qui détruit lentement, les usagers se sentent pris dans la double injonction d'une dépendance imposant la recherche de produit et l'urgence « vitale » d'y mettre fin.

\section{De l'exclusion à la marginalisation au sein même de la scène des produits}

L'usage du Subutex ${ }^{\circledR}$ est perçu à l'origine d'une accélération du processus de précarisation. Les modes de vie se sont enlisés dans l'univers de la rue jusqu'à atteindre des situations d'exclusion culminant dans la perte des liens familiaux ou affectifs (ruptures, décès). La pauvreté couplée à la lassitude, à l'épuisement des parcours et des corps tend à s'amplifier en une spirale infernale pour envahir l'ensemble de l'expérience de la personne : dégradation des conditions d'hygiène, altération profonde de l'estime de soi et de l'image corporelle, souffrance psychique et isolement.

La perception d'une vulnérabilité et d'une fragilisation croissantes s'accompagne de la restriction des ressources et des solidarités autrefois offertes par le milieu. Pour les usagers de 
«l'ancienne génération », les traitements de substitution ont déstructuré les anciens réseaux et les pratiques qui y étaient attachées. Marginalisés par ces «nouveaux toxicos » de la rue, maîtrisant mal les codes et n'adhérant pas aux systèmes de valeurs qui dominent aujourd'hui la scène de drogue, ils tendent à se replier au sein de micro-groupes rassemblant ceux qu'ils qualifient de « derniers survivants [de cette époque] ».

\section{Le sentiment de servir de cobaye}

Un phénomène de disqualification se joue à travers le double rejet des institutions (médecin, famille, police etc) et du monde de la rue. Le discrédit qui touche les usagers est extrême. Leur discours renvoie par ailleurs à une forme d'aliénation qui se caractérise par la perte de sens, l'isolement, et le sentiment d'absurdité d'être transformé en «chose» («On me traite comme une merde, j'ai l'impression de servir de cobaye », Julien, 38 ans). Aux prises avec l'enchaînement à un produit qui détruit alors qu'on l'avait supposé libérateur, le Subutex® devient objet de persécution. Ce sentiment d'instrumentalisation est largement partagé dans ce groupe. Tous prêtent aux pouvoirs publics et aux médecins des intentions malveillantes à l'égard des usagers de drogues; ils les soupçonnent d'utiliser les médicaments de substitution comme outil d'une politique de répression.

\subsection{Des modes d'appropriation pluriels du Subutex ${ }^{\circledR}$, instables dans le temps, et socialement différenciés}

\subsubsection{Des usages qui tendent à reproduire les fonctions d'une drogue}

L'étude a dégagé des situations contrastées dans le vécu de la dépendance correspondant à des modes d'appropriation et des itinéraires différenciés avec le Subutex®. Pour une partie de l'échantillon (profils 1 « ressource» et 2 «maintenance »), le Subutex® présente d'abord les caractéristiques d'un médicament ayant permis une amélioration significative des conditions de vie. Pour les uns (groupe 1 «ressource»), il agit comme support pharmacologique d'un 
processus plus global de normalisation des modes de vie, de redéfinition de soi, qui s'accompagne de changements majeurs dans l'utilisation des substances psychoactives. Le traitement doit se terminer avec une véritable sortie de la toxicomanie. Pour ceux qui s'inscrivent dans le profil 2, la dépendance constitue un facteur durable et peut-être définitif de vulnérabilité. Le traitement fait rempart aux risques persistants de rechutes et participe d'une qualité de vie améliorée. Dans ces cas de figure, on observe une prise régulée du Subutex ${ }^{\circledR}$. Chaque individu définit de façon autonome ses propres règles adaptant les doses et le nombre de prises quotidiennes. Les entretiens successifs sur une période de quelques mois et les récits des usagers des périodes antérieures indiquent le caractère toujours fragile des bénéfices tirés des traitements, même après de longues périodes d'amélioration. Pour l'autre moitié des répondants (profils 3 «drogue » et 4 «produit-piège »), le Subutex® est perçu comme une drogue qui s’est insérée de façon « fonctionnelle» dans les anciens systèmes de consommation et les modes de vie. Pour les uns (profil 3 «drogue légale »), il marque une transition vers des polyconsommations associant médicaments et alcool avec une chronicisation des états de dépendance. La disponibilité facile du Subutex ${ }^{\circledR}$ permet néanmoins des usages à moindre risques aussi bien en matière de délinquance que de risques pour la santé. Enfin, l'expérience du Subutex® fait franchir à certains (profil 4 «produit-piège) une nouvelle étape dans l'aggravation de la situation sociale et le mal-être et augmenter les dommages associés à des modes de consommations, de plus en plus chaotiques et compulsifs. L'échec est interprété comme une persécution perpétrée intentionnellement par les autorités qui assujettissent les usagers à un produit encore plus addictif et plus nocif pour le corps que les produits de rue, sans en apporter aucun des bénéfices, notamment le plaisir fugace de la drogue

\subsubsection{Des usages en miroir des situations sociales}

L'examen des situations sociales des usagers en fonction de leur positionnement dans les différents profils montre que les conditions de vie jouent un rôle majeur dans l'appropriation du 
Subutex ${ }^{\circledR}$ et les conduites de consommation. On observe en effet que les usagers correspondant aux profils 3 et 4 ont peu de qualification et qu'ils sont pour la très grande majorité en difficulté d'insertion sociale et professionnelle (chômage, minima sociaux, pas de logement personnel, alors que la plupart des usagers relevant des profils 1 et 2 ont une activité professionnelle, certains avec des emplois qualifiés et/ou permanents.

Tout se passe comme si la flexibilité du traitement favorisait la normalisation des modes de vie et renforçait le rôle favorable des ressources sociales chez les individus les mieux équipés socialement, tandis que pour ceux les plus en difficultés, l'échec avec le Subutex® se conjuguait à la précarité pour amplifier les vulnérabilités initiales ou consécutives aux années de toxicomanie (amendes impayées, dettes, peines suspendues à un sursis, vie à la rue, marquage des institutions répressives, altérations des relations sociales, faiblesse des expériences professionnelles, et états de santé dégradés) et pesant sur les capacités de s'en sortir.

\section{LE SUBTEX® : ENTRE DROGUE DE RUE ET MEDICAMENT}

Les traitements de substitution restent une pratique en marge du système de soins, suspectés d'entretenir la dépendance aux dépens de la collectivité, voire de générer des toxicomanes d'un nouveau type $\mathrm{e}^{6}$. Le Subutex ${ }^{\circledR}$ est devenu en valeur le $11^{\text {ème }}$ médicament vendu et remboursé par 1'assurance maladie en France et le $2^{\text {ème }}$ à Paris. Depuis 1996, année de mise sur le marché du Subutex®, le nombre d'usagers de drogue sous substitution ne cesse d'augmenter. Si certains indicateurs épidémiologiques (overdoses, contamination par le VIH) se sont améliorés et que l'usage de l'héroïne s'est considérablement réduit (O.F.D.T., 2003a), de nombreuses questions subsistent sur l'efficacité de cette stratégie en terme d'amélioration de la situation sociale et la qualité de vie des usagers de drogue d'une part, d'autre part sur l'existence d'un phénomène massif de détournement grâce à des prescriptions simultanées par des médicaments

\footnotetext{
${ }^{6}$ Les traitements de substitution en France ont fait successivement l'objet de recommandations de bonnes pratiques contre les mésusages (Afssaps-Anaes., 2004), d'une conférence de consensus (Conférence de consensus., juin 2004) et de modifications réglementaires visant à contrôler plus étroitement les pratiques de prescriptions.
} 
différents et d'effets négatifs liés à une fréquence élevée de l'injection du médicament broyé et dilué, entraînant abcès et problèmes veineux. L'étude visait à resituer ces phénomènes au sein de l'expérience des usagers avec les traitements et à rendre compte de la façon dont ils transforment la toxicomanie elle-même.

L'originalité de cette étude est d'avoir porté sur des populations aux profils individuels variés tant du point de vue des caractéristiques sociodémographiques que des contextes de vie et des modalités de traitements, dans Paris et dans sa proche banlieue. Les soignants qui ont introduit l'enquête auprès des usagers redoutant que l'entretien ne fragilise la relation thérapeutique ont pu exclure les usagers jugés plus «vulnérables » ou plus marginalisés. Le recrutement d'usagers par le biais des programmes à bas seuil (PES) dans lesquels les liens avec la structure ou les animateurs sont plus libres vient compenser cette possible sélection.

La réalisation des entretiens hors du cadre de soin, leur répétition, jouent en faveur de l'instauration d'un certain degré de confiance et limitent la sous-déclaration observée parmi les personnes traitées des usages de produits illicites (Falck et al., 1992; Sherman et Bigelow, 1992) et des conduites à risque (Desjarlais et al., 1996). D'ailleurs, après un premier entretien où l'interviewé tend souvent à privilégier les événements et situations qui correspondent à l'image qu'il veut donner de lui-même, les récits ultérieurs dégagent les éléments plus solides des parcours : les événements survenus entre les entretiens prennent place dans une histoire, sont mis en perspective et les biais de mémorisation sont ainsi atténués ou corrigés. En outre, les entretiens se sont centrés sur la vie quotidienne sans focaliser sur l'observance ou sur la consommation de drogues illicites.

Cependant les récits portent sur une courte séquence de temps ( 4 mois environ) au sein d'une histoire avec les produits souvent de plus de 10 ans, marquée par une forte instabilité. Celle-ci est apparue d'ailleurs dans la série des entretiens où les usagers ont pu rendre compte de rapports différents au traitement et à l'usage des produits. Les profils constituent des " photographies », chaque personne interrogée étant reclassée dans le profil qui caractérise le 
mieux sa situation au cours de la période des entretiens. Toutefois il serait réducteur de «fixer » les usagers dans un « profil type » (Neale, 1999a; Fischer et al., 2002a).

L'analyse faite ici des récits des usagers sous Subutex® dans leurs attentes, leurs usages du médicament, leurs perceptions du traitement, les bénéfices et difficultés face à la substitution recoupe pour une grande part les observations faites avec la méthadone dans d'autres pays. C'est le cas notamment pour ce qui a trait à la diversité des rapports au traitement, aux changements au cours de la prise en charge allant d'une reprise de contrôle du mode de vie à une détérioration continue, à la coexistence chez les mêmes individus de satisfactions et d'insatisfactions, et aux fluctuations des situations (Gayre et Richard, 2000).

Jusqu'à maintenant où la méthadone et son encadrement strict dominent l'offre de substitution dans les pays occidentaux, il était difficile de démêler dans les observations et les analyses ce qui tenait à un régime étroitement contrôlé avec un produit particulier de ce qui relevait des effets plus larges de la substitution d'un médicament à une drogue. Les observations faites en France avec un produit différent et une grande flexibilité d'accès apporte un éclairage nouveau.

\section{Le Subutex ${ }^{\circledR}$ : un produit addictif et pauvre en sensations}

L'observation de modes d'appropriation similaires du Subutex ${ }^{\circledR}$ et de la méthadone tient tout d'abord à certaines propriétés pharmacologiques communes qui génèrent des effets différents de ceux de l'héroïne de rue : longue durée d'action, blocage du syndrome de manque, effet sur le « craving », effets secondaires (Amato et al., 2004; Ling et Wesson, 2003; Mattick et al., 2003). Cependant, par ses propriétés spécifiques, notamment la faiblesse de la sensation d'euphorie et du « flash », la buprénorphine [par rapport à la méthadone], peut améliorer le bénéfice chez ceux qui cherchent à normaliser leur style de vie, à éprouver des sensations physiques et émotionnelles dégagées de l'effet de l'héroïne, et qui perçoivent alors le Subutex® comme un simple médicament. C'est le cas notamment chez ceux qui ont déjà une bonne insertion sociale ou qui 
intègrent le médicament de substitution à la gestion de leur santé mise à mal par des pathologies plus graves. À l'inverse, on peut faire l'hypothèse que l'insatisfaction liée à cette pauvreté des effets recherchés majore le recours à d'autres substances, (médicaments, cocaïne ou alcool) ou la pratique de l'injection, notamment parmi ceux pour qui cette recherche de sensation est nécessaire ou revendiquée. Ce phénomène qui associe le produit et ses conditions de dispensation est relevé par de nombreux auteurs qui dénoncent la méthadone comme un dispositif aggravant la situation des toxicomanes (Bourgois, 2000; Johnson et Friedman, 1993; Koester et al., 1999; Rosenbaum et Murphy, 1984; Uchtenhagen et al., 1997). Les consommations incontrôlées - qui culminent les jours d'aisance financière, en particulier de paiement des allocations d'assistance résulteraient de l'absence de plaisir dans la prise de méthadone, des sensations persistantes de manque en raison de doses insuffisantes, de la pénibilité des effets secondaires, et des contraintes rigides de son encadrement (Bourgois et al., 1997). Le profil 4 « produit-piège » correspond à ce processus : on y retrouve l'addiction au Subutex ${ }^{\circledR}$, la permanence des sensations de manque, l'insuffisance des effets qui entretiennent une consommation désordonnée de produits, notamment les benzodiazépines, avec une totale perte de contrôle de la conduite. C'est aussi dans ce registre qu'on doit situer l'injection.

\section{Un encadrement du traitement qui offre une grande autonomie}

Le principe qui préside aux traitements de substitution est de déplacer le cadre de la dépendance de la scène de la drogue au cabinet médical ou au centre spécialisé, et de remplacer une drogue de rue par un «médicament». Ce déplacement est analysé par Bourgois (2000) comme une disciplinarisation des toxicomanes par la médecine: ils sont soumis par la substitution au pouvoir de la médecine et à ses règles de façon beaucoup plus étroite qu'ils ne le sont au dealer et plus généralement aux normes de la rue. Cette soumission opère à travers les règles des centres de soins : enregistrement obligatoire, venue quotidienne, contrôle visuel de la 
prise du médicament ou des prélèvements d'urines, sanctions en fonction du résultat des tests urinaires (Neale, 1999a; Gayre et Richard, 2000).

A l'inverse de ce contrôle rigide, la flexibilité du système français en matière de traitement de substitution avec le Subutex ${ }^{\circledR}$ favorise l'autonomie des usagers dans toutes les dimensions du traitement (choix du praticien, choix du pharmacien, horaire des consultations, horaires et modes des prises puisque la personne a ses médicaments pour quelques jours ou quelques semaines). On le voit dans les profils 1 et 2. En revanche dans les situations d'échec que l'on retrouve dans les profils 3 et 4 , la disponibilité quasi sans limite du médicament rend encore plus difficile la gestion du produit au quotidien. L'absence de contrôle conduit alors à intégrer à la logique du monde de la drogue tant le médecin ou le pharmacien que le produit de substitution.

\section{L'injection du médicament broyé : une composante autonome dans la dépendance}

La problématique de l'injection a en effet une place centrale dans la contestation de la valeur thérapeutique du Subutex ${ }^{\circledR}$ en France. L’injection du médicament $(11 \%$ à $48 \%$ des utilisateurs selon les études (O.F.D.T., 2004), porte atteinte au crédit d'un traitement mis en place dans le contexte d'urgence du sida. En France, la forme galénique en comprimé, facile à broyer et à diluer dans un petit volume liquide et injectable malgré ses risques, a été montrée comme un déterminant du maintien de l'injection chez les personnes sous Subutex (Guichard et al., 2003). Ailleurs où la méthadone domine, son injection est rare (Humeniuk et al., 2003). L'injection est un marqueur de sévérité connu de la toxicomanie, phénomène retrouvé dans plusieurs études menées en France montrant que l'injection du produit de substitution est associée à une consommation intense et chaotique du Subutex ${ }^{\circledR}$ en combinaison d'autres substances, à la recherche de « défonce » et à une forte désinsertion sociale (Vidal-Trecan et al., 2003; Varescon et al., 2002; Courty, 2003; Blanchon et al., 2003; Brunelle, 2003).

Dans les travaux menés sur la pratique d'injection, celle-ci apparaît de plus en plus comme une dimension en partie autonome au sein du phénomène de dépendance à l'héroöne et 
pas seulement comme un moyen de maximiser les effets (McBride et al., 2001; Fischer et al., 2002b). Sa persistance ou son arrêt au cours du traitement n'est pas directement associé à l'évolution de l'usage de l'héroïne (Kelley et Chitwood, 2004; Langendam et al., 2000). Cette autonomie relative de ces deux phénomènes apparaît également dans notre étude. En effet, dans les profils 1 et 2, l'arrêt de l'injection est survenu progressivement au fil de l'itinéraire thérapeutique; l'ancienneté de cet abandon favorise l'appropriation du Subutex ${ }^{\circledR}$ comme une ressource pour s'en sortir. Ces résultats incitent à la prendre en compte comme un enjeu en soi dans la prise en charge et à considérer la cessation de l'injection comme un de ses objectifs.

\section{Les déterminants sociaux des modes d'appropriation du Subutex ${ }^{\circledR}$}

Comme on l'a vu, la souplesse du dispositif du Subutex® quant à ses modalités d'accès ou de gestion de la prescription favorise une relative autonomie des usagers au cours du suivi médical. La prise en compte des caractéristiques personnelles, notamment sociales, des personnes interrogées, confirme que la situation sociale et le contexte de vie jouent un rôle majeur dans l'appropriation et la régulation des usages d'une substance (Norman et Zinberg, 1980; Simpson et al., 2000).

Cette flexibilité profite davantage à ceux qui bénéficient de ressources économiques et d'un capital social ${ }^{7}$ suffisants. Pour les plus vulnérables en revanche, elle favorise une accessibilité sans véritable limite au produit, l'augmentation des quantités consommées sans satisfaction, conduisant à des consommations multiples, à des injections de plus en plus néfastes, au maintien dans l'univers des drogues de rue (Duprez et Kokoreff, 2000; Bouhnik et al., 1999; Bouhnik et Touzé, 2001). Pour autant, on observe comme l'a déjà montré A. Lovell (2001), que les usagers les plus fragilisés peuvent trouver dans l'accès aisé au Subutex ${ }^{\circledR}$ une forme de réponse face à des problèmes juridiques, économiques ou sociaux, même quand il est injecté.

\footnotetext{
${ }^{7}$ Par « capital social», on entend l'ensemble des relations (parents, amis, collègues, par exemple) actuelles ou potentielles qu'un individu est en mesure de mobiliser pour agir. Il s'agit plus simplement de ce que Lin (1995) appelle les « ressources sociales » d'un individu.
} 


\section{Une image de soi complexe porteuse du stigmate de la substitution}

L'analyse des entretiens a mis en évidence le poids persistant de l'image péjorative du toxicomane comme un obstacle même dans les cas les plus favorables à l'adoption d'un rôle de personne traitée. Dans les études menées aux Etats-Unis ou au Royaume uni, le stigmate qui pèse sur l'utilisateur de méthadone est d'abord mis en relation avec le caractère infantilisant des services de soins évoqué précédemment (Bourgois, 2000; Fischer et al., 2002a; Hunt et Rosenbaum, 1998; Hunt et al., 1985; Lovejoy et al., 1995; Neale, 1999b). En France, en dépit d'un dispositif intégré à la médecine générale, les traitements de substitution n'ont pas acquis le statut de traitement ordinaire. L'existence d'un marché de rue et d'un encadrement jugé laxiste, y compris par une partie des usagers, entretient une représentation négative de l'usager. Le sevrage «de tout produit» reste pour beaucoup, les usagers, leur entourage voire de nombreux professionnels, le seul mode d'affranchissement du passé de toxicomane. Dans le milieu même des usagers de drogues de rue, le consommateur d'un produit de substitution est perçu comme l'antithèse du « righteous dope friend », figure valorisée d'indépendance et de résistance aux lois et aux institutions (Koester et al., 1999; Bourgois, 2000). L’usager traité, déjà « désaffilié » perd ses liens avec la culture de rue et se trouverait ainsi doublement exclu comme l'a déjà montré $\mathrm{R}$. Castel (1998) dans une analyse retraçant les processus de «sortie de la toxicomanie». A l'extrême comme dans le profil 4, l'usager se sent devenu l'objet d'une expérience déshumanisée en se représentant comme un cobaye.

Ainsi la recherche d'une banalisation du traitement de substitution par son intégration au régime de la médecine générale ne suffit pas à faire exister la substitution comme un traitement comme un autre. De fait, la porosité entre le monde de la drogue de rue et le monde du traitement que souligne Agar et al. (2001) rend plus difficile l'émergence d'une identité de personne soignée. Les produits de substitution sont croisés très tôt dans les trajectoires, et l'usage de rue des médicaments précède l'entrée en traitement pour la quasi-totalité des toxicomanes interrogés 
dans l'enquête Aides-Inserm (2001). La régularisation de cette consommation qui s'opère avec le traitement baisse de façon très forte l'usage des substances illégales et permet l'adoption de nouveaux modes de consommation. La faiblesse des contrôles dans le régime du Subutex ${ }^{\circledR}$ réduit les ruptures, lisse les trajectoires et évite le phénomène de «porte tournante » qu'on observe dans le système strict de la méthadone (Koester et al., 1999). Le traitement de substitution devient ainsi une des modalités de la «carrière » de toxicomanie qui alterne périodes de consommations sans frein, traitements, rémissions et rechutes (Hser et al., 1993; Nurco et al., 1981; Hser et al., 2001), échecs et avancées.

Dans le paysage des politiques de santé publique face à la toxicomanie, la France fait figure de retardataire et de franc-tireur. Au niveau collectif, les résultats sont tangibles avec la quasi-disparition des nouvelles contaminations par le VIH, la baisse des overdoses et celle de la consommation d'héroïne. Notre recherche éclaire la dimension individuelle de l'impact de cette stratégie. On y retrouve des effets marqués de réduction des risques qui peuvent éviter les pertes de contrôle exposant à de plus grands risques. Le bénéfice pourtant est loin d'être homogène. Les mieux dotés socialement reconquièrent leur autonomie par rapport à l'astreinte de la dépendance tandis que pour les autres s'accentue l'engrenage de la marginalisation. En quelque sorte une majorité peut tirer du système un bénéfice sanitaire mais aux extrêmes du spectre social les effets, positifs ou négatifs, sont amplifiés. 


\section{BIBLIOGRAPHIE:}

Afssaps-Anaes., 2004, Réduire les mauvaises utilisations des médicaments de substitution des opiacés: recommandations pour la pratique clinique, Saint-Denis, Afssaps-Anaes, $18 \mathrm{p}$

Agar M., Bourgois P., French J. et Murdoch O., 2001, Buprenorphine: "field trials" of a new drug, Qualitative Health Research 2 11, 1, 69-84.

Aides-Inserm., 2001, Attentes des usagers de drogue concernant les traitements de substitution: expérience, satisfaction, effets recherchés, effets redoutés, Paris, Aides/Inserm, $56 \mathrm{p}$.

Amato L., Davoli M., Ferri M., Gowing L. et Perucci C. A., 2004, Effectiveness of interventions on opiate withdrawal treatment: an overview of systematic reviews, Drug Alcohol Depend 2 73, 3, 219-26.

Beschner G. M. et Walters J. M., 1985, Just another habit? The heroin users' perspective. In: Hanson B., Beschner G. M., Walters J. M. et Bovelle E., eds, Life with heroin: voices from the inner city, Lexington MA, Lexington Books, 155-173

Blanchon T., Boissonnas A., Vareseon I. et Vidal-Trecan G., 2003, Homelessness and highdosage buprenorphine misuse, Subst Use Misuse 2 38, 3-6, 429-42.

Bouhnik P., Jacob E., Maillard I. et Touzé S., 1999, L'amplification des risques chez les usagers de drogues précarisés, Paris, Ministère de l'emploi et de la solidarité- Direction générale de la santé- Ministère de la justice- Direction de l'administration pénitentiaire / Resscom (Recherches et évaluations sociologiques sur le social, la santé et les actions communautaires), $155 \mathrm{p}$

Bouhnik P. et Touzé S., 2001, Précarisation et consommations de drogues illicites: amplification des prises de risques à l'ère de la substitution. In: Joubert M., Chauvin P., Facy F. et Ringa V., eds, Précarisation, risque et santé, Paris, Inserm, 395-412.

Bourgois P., 2000, Disciplining addictions: The bio-politics of methadone and heroin in the United States, Culture, Medicine and Psychiatry, 24, 2, 165-195.

Bourgois P., Lettiere M. et Quesada J., 1997, Social misery and the sanctions of substance abuse: confronting HIV risk among homeless heroin addicts in San Francisco, Social Problems $44,2,155-173$

Brown B. S., Benn G. J. et Jansen D. R., 1975, Methadone maintenance: some client opinions, Am J Psychiatry 2 132, 6, 623-6.

Brunelle E., 2003, Analysis of drug consumption among ambulatory patients in a drug agency of Northern France (Lens), Ann Med Interne (Paris) 154 Spec No 1, S15-23.

Castel R., 1998, Les sorties de la toxicomanie, Fribourg, Editions universitaires Fribourg Suisse.

Conférence de consensus., juin 2004, Textes des recommandations. Stratégies thérapeutiques pour les personnes dépendantes aux opiacés: place des traitements de substitution, Lyon, Anaes-FFA, $40 \mathrm{p}$.

Courty P., 2003, High dosage buprenorphine and injection practices. A study of 303 patients, Ann Med Interne (Paris) 2154 Spec No 1, S35-45.

Desjarlais D. C., Friedmann P., Hagan H. et Friedman S. R., 1996, The prospective effect of AIDS-related behavioral change among injection drug users: a cross-national study, American Journal of Public Health $286,1780-1785$.

Duprez D. et Kokoreff M., 2000, Les carrières de consommation. In: Duprez D. et Kokoreff M., eds, Les mondes de la drogue, Paris, Editions Odile Jacob, 111-191.

Falck R., Siegal H. A., Forney M. A., Wang J. et Carlson R. G., 1992, Self-reported use of opiates and cocaïne, Journal of Drug Issues, 22, 823-832

Fischer B., Chin A. T., Kuo I., Kirst M. et Vlahov D., 2002a, Canadian illicit opiate users' views on methadone and other opiate prescription treatment: an exploratory qualitative study, Subst Use Misuse 2 37, 4, 495-522.

Fischer B., Rehm J., Kirst M., Casas M., Hall W., Krausz M., Metrebian N., Reggers J., Uchtenhagen A., van den Brink W. et van Ree J. M., 2002b, Heroin-assisted treatment as 
a response to the public health problem of opiate dependence, European Journal of Public Health $_{2}$ 12, 3, 228-34.

Gayre C. et Richard H., 2000, Service users' and providers' perceptions of a methadone maintenance program: a Queensland qualitative pilot study, Queensland, 1-150.

Gold M. L., Sorensen J. L., McCanlies N., Trier M. et Dlugosch G., 1988, Tapering from methadone maintenance: attitudes of clients and staff, $J$ Subst Abuse Treat ${ }_{2} 5,1,37-44$.

Guichard A., Lert F., AIDES., Brodeur J. M., Richard L., Benigeri M. et Zunzunegui M. V., 2003, Illicit Drug Use and Injection Practices among Drug Users on Methadone and Buprenorphine Maintenance Treatment in France, Addiction 2 98, 1585-1597.

Hser Y. I., Anglin D. et Powers K., 1993, A 24-year follow-up of California narcotics addicts, Arch Gen Psychiatry, 50, 7, 577-84.

Hser Y. I., Hoffman V., Grella C. E. et Anglin M. D., 2001, A 33-year follow-up of narcotics addicts, Arch Gen Psychiatry, 58, 5, 503-8.

Humeniuk R., Ali R., McGregor C. et Darke S., 2003, Prevalence and correlates of intravenous methadone syrup administration in Adelaide, Australia, Addiction ${ }_{2}$ 98, 4, 413-8.

Hunt D. E., Lipton D. S., Goldsmith D. S., Strug D. L. et Spunt B., 1985, "It takes your heart": the image of methadone maintenance in the addict world and its effect on recruitment into treatment, Int $J$ Addict, 20, 11-12, 1751-71.

Hunt G. et Rosenbaum M., 1998, "Hustling" within the clinic: consumer perspectives on methadone maintenance treatment. In: Inciardi J. A. et Harrison L. D., eds, Heroin in the age of crack-cocaine, London, Sage Publ., 188-214.

Inserm, 1998, Evaluer la mise à disposition du Subutex pour la prise en charge des usagers de drogue. Synthèse rapide de la littérature et des données disponibles et propositions pour un programme de recherche, Saint-Maurice, Rapport Inserm U88 (Sous la direction de France Lert), $96 \mathrm{p}$.

Johnson P. et Friedman J., 1993, Social versus physiological motives in the drug careers of methadone clinics clients, Deviant Behavior 2 14, 23-42.

Kelley M. S. et Chitwood D. D., 2004, Effects of drug treatment for heroin sniffers: a protective factor against moving to injection?, Soc Sci Med 2 58, 10, 2083-92.

Koester S., Anderson K. et Hoffer L., 1999, Active heroin injectors' perceptions and use of methadone maintenance treatment: cynical performance or self-prescribed risk reduction?, Subst Use Misuse 2 34, 14, 2135-53.

Langendam M. W., van Brussel G. H., Coutinho R. A. et van Ameijden E. J., 2000, Methadone maintenance and cessation of injecting drug use: results from the Amsterdam Cohort Study, Addiction 2 95, 4, 591-600.

Lin N., 1995, Les ressources sociales: une théorie du capital social, Revue Française de Sociologie $36,685-704$.

Ling W. et Wesson D. R., 2003, Clinical efficacy of buprenorphine: comparisons to methadone and placebo, Drug Alcohol Depend 2 70, 2 Supp1, S49-57.

Lovejoy M., Rosenblum A., Magura S., Foote J., Handelsman L. et Stimmel B., 1995, Patients' perspective on the process of change in substance abuse treatment, $J$ Subst Abuse Treat ${ }_{2}$ $12,4,269-82$.

Lovell A., 2001, Ordonner les risques: l'individu et le pharmaco-sociatif face à l'injection de drogues. In: Fassin D., eds, Critique de la santé publique, Paris.

Magura S. et Rosenblum A., 2001, Leaving methadone treatment: lessons learned, lessons forgotten, lessons ignored, Mt Sinai J Med $268,1,62-74$.

Mattick R. P., Ali R., White J. M., O'Brien S., Wolk S. et Danz C., 2003, Buprenorphine versus methadone maintenance therapy: a randomized double-blind trial with 405 opioiddependent patients, Addiction 2 98, 4, 441-52.

McBride A. J., Pates R. M., Arnold K. et Ball N., 2001, Needle fixation, the drug user's perspective: a qualitative study, Addiction 2 96, 7, 1049-58. 
Montagne M., 1998, Philosophies of drug giving and drug taking, Journal of Drug Issues 218 , $139-284$

Montagne M., 2002, Appreciating the user's perspective: listening to the "methadonians", Subst Use Misuse 2 37, 4, 565-70

Neale J., 1999a, Drug users' views of substitute prescribing conditions, International Journal of Drug and Policy, 10, 247-258.

Neale J., 1999b, Understanding drug-users clients' views of substitute prescribing, Br J Soc Work $29,1,127-145$.

Newman R., 1996, The promises and pitfalls of methadone treatment, International Journal of Drug and Policy, 7, 66-67.

Norman E. et Zinberg N. E., 1980, The social setting as a control mechanism in intoxicant use. In: Wallenstein Pearson H., eds, Theories on drug abuse, Rockville, NIDA, 30.

Nurco D. N., Cisin I. H. et Balter M. B., 1981, Addict careers. I. A new typology, Int J Addict, $16,8,1305-25$.

O.F.D.T., 2003a, Phénomènes émergents liés aux drogues en France en 2001. Rapport Trend 2002, Paris, OFDT, 287 p.

O.F.D.T., 2003b, Substitution aux opiacés: Synthèse des informations disponibles de 1996 à 2001 en France, Paris, OFDT, 80 p.

O.F.D.T., 2004, Les traitements de substitution en France: résultats récents 2004 . N³7. Tendances, Paris, OFDT, $8 \mathrm{p}$.

Ogien A., 1995, Sociologie de la déviance, Paris, Armand Colin.

Rosenbaum M. et Murphy S., 1981, Getting the treatment: recycling women addicts, $J$ Psychoactive Drugs, 13, 1, 1-13.

Rosenbaum M. et Murphy S., 1984, Always a junkie?: the arduous task of getting off methadone, Journal of Drug Issue 2 16, 4, 527-552.

Rosenblum A., Magura S. et Joseph H., 1991, Ambivalence toward methadone treatment among intravenous drug users, $J$ Psychoactive Drugs 23 , 1, 21-7.

Seidman I., 1998, Interviewing as qualitative research, New York, Teachers College Press.

Sherman M. F. et Bigelow G. E., 1992, Validity of patients' self-reported drug use as a function of treatment status, Drug and Alcohol Dependence, 30, 1-11.

Simpson D. D., Joe G. W., Greener J. M. et Rowan-Szal G. A., 2000, Modeling year 1 outcomes with treatment process and post-treatment social influences, Subst Use Misuse ${ }_{2}$ 35, 12-14, 1911-30

Trostle J. A., 1997, The history and meaning of patient compliance as an ideology. In: Gochman D., eds, Handbook of health behavior research: provider determinants, New York, Plenum, 2, 49-62.

Uchtenhagen A., Gutzwiller F. et Dobler-Mikola A., 1997, Essais de prescription médcale de stupéfiants. Rapport de synthèse PROVE, Zurich, Institut de Médecine Sociale et Préventive, $162 \mathrm{p}$.

Varescon I., Vidal-Trecan G., Nabet N. et Boissonnas A., 2002, Buprenorphine abuse: high dose intravenous administration of buprenorphine, Encephale 2 28, 5 Pt 1, 397-402.

Vidal-Trecan G., Varescon I., Nabet N. et Boissonnas A., 2003, Intravenous use of prescribed sublingual buprenorphine tablets by drug users receiving maintenance therapy in France, Drug Alcohol Depend 269,2 , 175-81. 


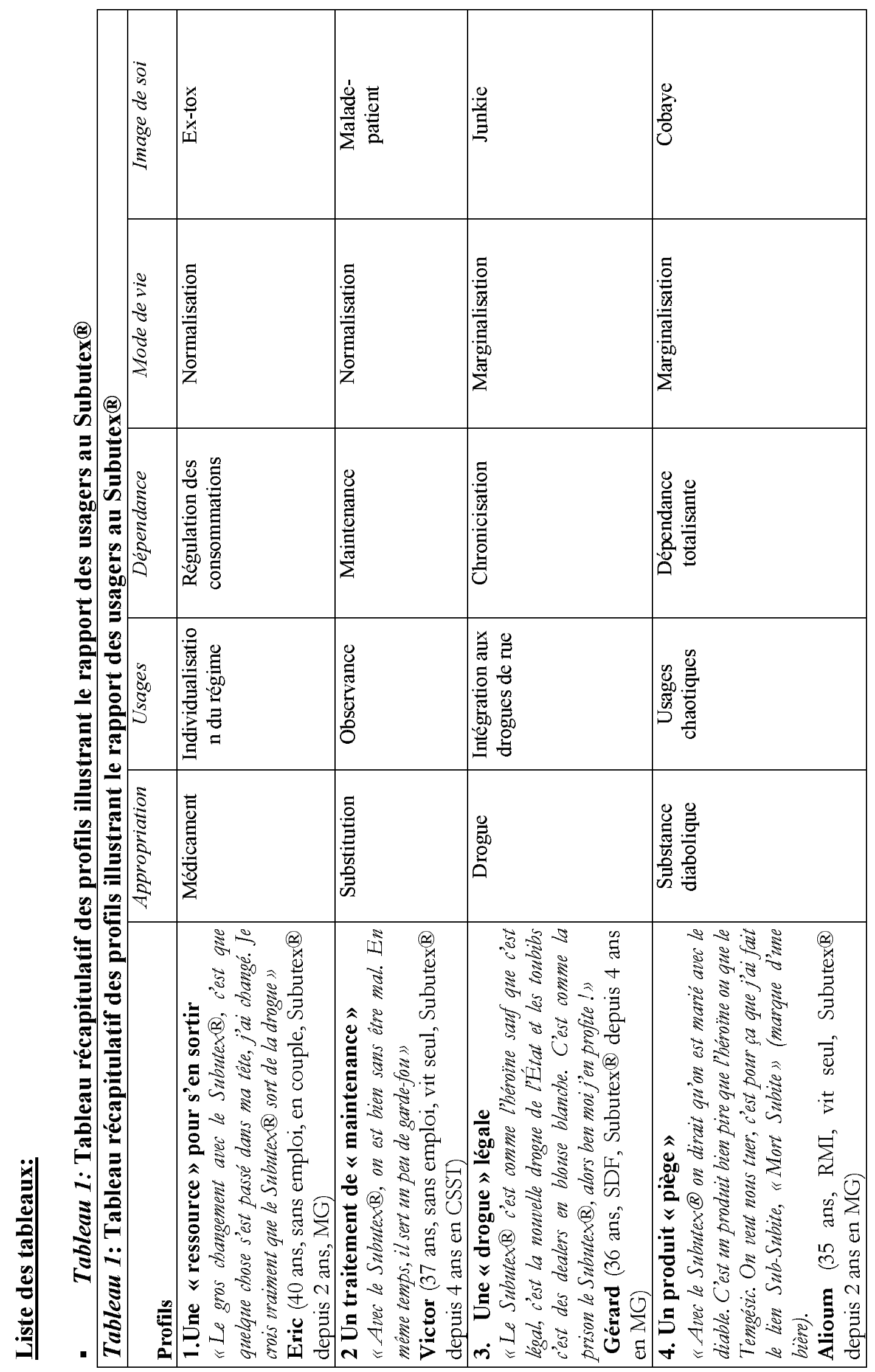


